\title{
A Reliable and Efficient Path Discovery Method for Mobile Sink based Wireless Sensor Networks
}

\author{
Elie T. Fute \\ Department of Mathematics and \\ Computer Science, University of \\ Dschang \\ Department of Computer \\ Engineering, \\ University of Buea \\ Cameroon
}

\author{
Aline Z. Tsague \\ Department of Computer \\ Engineering, \\ University of Buea \\ Department of Mathematics and \\ Computer Science, University of \\ Dschang, \\ Cameroon
}

\author{
Emmanuel Tonye \\ Department of Electrical and \\ Telecommunication Engineering, \\ University of Yaounde I, \\ Cameroon
}

\begin{abstract}
Wireless sensor networks (WSNs) are made of autonomous sensor nodes distributed into a given physical environment to monitor the events and communicate with a base station or sink. Data gathering therefore appears to be a major objective of sensor nodes in WSNs. Mobile sinks (MS) have been widely used for this purpose as they implicitly provide loadbalancing and help achieving uniform energy-consumption across the network. Several path discovery schemes for mobile sink have been proposed in the literature. However most of these solutions do not leverage the fact that the process of identification/selection of visit points can be executed as many times as needed, in order to balance the energy consumption at these points, this without much expending network resources. Generally these points are referred as cluster heads and the procedure of establishing such a cluster topology is called clustering. In this paper, is presented an efficient path discovery scheme which not only addresses the above mentioned issue, but also determines target points at which the MS stops in order to ensure reliable communication during data transfer The proposed scheme employs well known protocols from the literature namely the instantaneous clustering algorithm (ICP) for WSN, whose main objective is to minimize the total time consumption of clustering. The circumference visit method is utilized to determine the target points which form the path of the MS. With the performance analysis performed, it's shown that the proposed method increases network lifetime, by reducing the amount of data transmissions and time consumption of clustering.
\end{abstract}

\section{General Terms}

Data collection, Mobile sink, Wireless sensor network.

\section{Keywords}

Clustering, Coverage, Data gathering, Mobile sink, Sensor network.

\section{INTRODUCTION}

Advances under the miniaturization of electronic components, combined with the standardization of wireless communications and the concern to improve the quality of life for humans had led to the advent of wireless sensor network (WSN) technology [1, 2]. Since their inception, sensor-based wireless communication networks have experienced a growing success within the scientific and industrial communities. Thanks to its numerous advantages (cheap installation and maintenance costs, fast and high data transmission speed, easy access, etc), this technology has been able to institute itself as a mainstay in current network architectures, particularly for the Internet of Things (IoT). Indeed, through the use of sensors, any physical infrastructure (vehicles, smart phones, home and offices appliances, etc) can be closely associated with information and communication technologies [4, 5].

A wireless sensor network (WSN) consists of a set of sensor nodes which are battery-powered resource constrained devices, deployed in a more or less random manner in order to monitor and control an environment, known as the sensor field or sensing area $[2,3,4]$. The sensor nodes cooperatively sense various events in their surrounding environment and report back to a base station [3,5]. As for the sensor field, it can be taken in the air (flying sensor networks), on or under the ground (wireless underground sensor networks), within water (wireless underwater sensor networks), etc. Thus, according to the application area, the sensor field can take various natures and shapes. For instance in medical applications [6], the sensing area represents the human body, on which sensors are placed to collect relevant physiological parameters such as heart rate, blood pressure and blood sugar, which are very sensitive and important because these data are the basis of clinical diagnostics. In environmental and military applications, the sensing area represents a geographical location well defined and delimited, which could be found in the air, on or under the ground and in the water. Such locations include the premises of a building, part of a forest, part of lake, an airport, a plantation, etc. The sensing area can further be classified as attended or unattended, based on whether or not it eases external intervention. More particularly in unattended wireless sensor networks (UWSN), a mobile sink (MS) regularly visits the network to collect data from sensor nodes.

Indeed, data gathering is the main objective of sensor nodes. The sensors periodically sense the data from their surrounding environment, process it and transmit it to the base station or sink $[3,5,8]$ as depicted on Figure 1 . The frequency of reporting the data and the number of sensors which report the data depends on the particular application as WSNs can be applied for a varied range of applications. Applications can be classified in four categories, according to how data exchange is initiated. The first is event-driven applications, in which data exchanges start when some event happens. The second is query-driven applications, in which exchange only starts when a specific node sends a query. This specific node can be the sink node in the case of static wireless sensor networks (WSNs). The third is time-triggered applications, in which exchanges happen at predefined instants. Finally, some 
applications such as live video transmission require data to continuously flow through the network. Thus data gathering involves systematically collecting the sensed data from multiple sensors and transmitting the data to the base station for further processing [8] as shown on Figure 1.

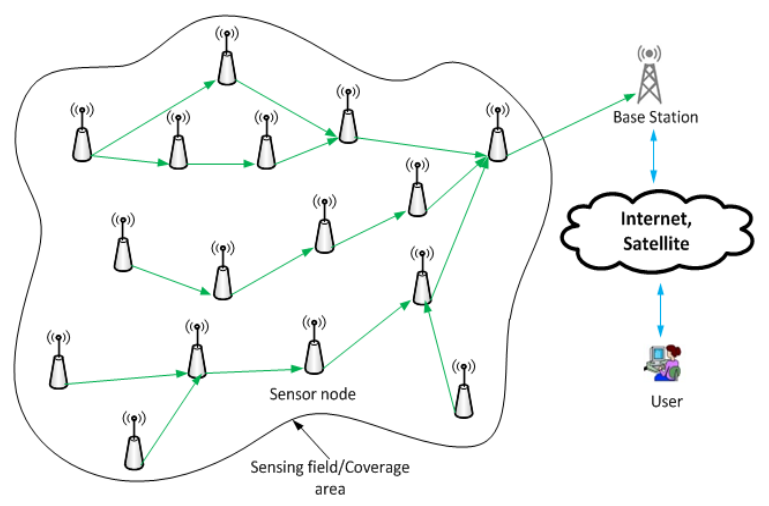

Figure 1: A WSN with a flat structure

Yet, the network might not be fully connected due to sensor nodes being static and sparsely deployed, generating large inter-sensor distances and making it impossible for sensor nodes to send data to the sink by multi-hop communication [9]. Also in the case of the mobile sink visiting all sensor nodes in the network, results in achieving coverage and data collection efficiency with high delay and high energy consumption. A mobile sink is employed in such cases to collect data from individual sensor nodes $[3,7,8]$, which have been assigned to hold data from other neighboring nodes. The use of a mobile sink is also to provide load balancing and help achieve uniform energy-consumption across the network [12], thus solving the sink neighborhood problem [7,9] in which nodes located within a single hop of the sink (static) node expend more energy than other nodes in the network. This problem can further cause a partition in the network and reduce sensing coverage, resulting in the premature expiry of the network. Consequently, relevant sensor nodes retain their data for retrieval by the mobile sink during its visits.

Yet several design issues need to be considered when implementing mobile sink for WSNs so as to avoid an overhead in terms of energy consumption and packet delays. These include the following:

Knowledge of target nodes' locations by the MS

Nearness of the MS with the target node

Minimal total round trip distance

Sufficient time/opportunity to exchange data with target node

In this paper, an efficient method to discover the optimal path for a mobile sink that visit sensor nodes in a wireless sensor network is presented. The sensors nodes are heterogeneous with variable communication ranges. The method utilizes the instantaneous clustering algorithm (ICP) for WSN in [10] to select and determine with respect to time and energy efficiency the locations of the target nodes along the tour path of the mobile sink. Indeed the need for a clustering technique is very useful in that it determines the visit points from which data will be collected by the MS rather than visiting all the nodes. In addition, for self-organizing purposes and without stopping the sensing tasks, a WSN usually demands several times of reclustering in its life-time. The ICP protocol is used for it efficiency in terms of time and energy. From these perspectives, a fast clustering is not only significant in WSNs (especially in mission-critical applications such as military surveillance and disaster relief) but also indicates a lightweight clustering process with low transmission overhead, and has the potential to cut down the total energy consumption [10].

The method also employs the circumference visit method for data collection in order to achieve reliable communication between target node and MS. Thus the power required by the sensor to transmit data to the TS is the lowest [9].

The remainder of this paper is organized as follows. Section 2 provides a brief survey of related works. Some preliminaries are introduced in section 3. In Section 4, the proposed scheme is described in detail. Section 5 describes the experimental results and analysis of the proposed scheme. Finally, concluding remarks and future prospects are provided in Section 6.

\section{RELATED WORK}

The use of mobile sinks in WSNs for the purpose of data collection has presented lots of benefit as they implicitly provide load-balancing and help achieving uniform energyconsumption across the network. There are several energyefficient and intelligent path discovery schemes for mobile sink which have been proposed in the literature. This section reviews the work done on path discovery for mobile sink and with reliable communication during data collection.

In [7] a scheme for secure data transmission and balanced energy consumption in an unattended wireless sensor network (UWSN) is presented. The network is comprised of multiple static source nodes and a mobile sink in the presence of adversaries. The proposed system has three phases: the identification of data collection points (called convex nodes), the path planning by the mobile sink, and secure data transmission. The convex nodes are determined using the energy-aware convex hull algorithm and are all found around the edges of the network boundary. The convex nodes are used as data collection points in order to reduce the time required to cover the network by the mobile sink. Although this system can be suitable for some particular type of application scenario (such as hostile battlefield surveillance where nodes monitor the possible placement of landmines by an intruder), it still presents some breakdowns. The network architecture used is flat and energy consumption is not balanced as the convex nodes deplete their energy quickly.

The work in [9] introduces a novel technique for better communication between the travelling sink (TS) and the sensors. Their method utilizes a modified travelling salesman problem in which the traveler is the mobile sink (called travelling sink) and the targets are the sensor nodes. The sensor nodes are heterogeneous with different communication ranges. In the proposed work, the mobile sink has to visit every sensor node in the network to collect the data from that sensor. Yet this operation is very costly in terms of time and total round trip distance, especially if the network is dense. Moreover the TS travels along the edges (called chords) connecting two points of the circles that represent the communication range of the sensor nodes. Thus the travel path of the TS is made of series of alternating chords and connecting links in between them. To determine the optimal locations of these chords along the tour path of the TS, the authors proposed three methods namely constant subtended angle chord lengths, fixed chord lengths and variable chord lengths. 
In [13], the authors present a new algorithm that finds the shortest round trip rectilinear route covering some specified nodes in a grid based Wireless Sensor Network with a moving sink. In a rectilinear route, the MS is restricted to travel in two directions only: horizontally or vertically along the connecting edges. In the proposed scheme, the network is considered as a graph with the grid points being the vertices of the graph and the lines joining the grid points being the edges. The optimal edge set that forms the target path is determined using the binary integer programming in which both the shortest path and the minimum bend criterion are met simultaneously. Thus, the method selects the path which has the least number of corners (bends) when more than one equal length shortest round trip paths are found.

In an earlier work proposed in [14], a residual energy based clustering and data gathering protocol for wireless sensor networks with delay constraints is presented. The network is first subdivided into clusters using an energy-efficient multihop clustering algorithm, taking into account the orientation of the sensor nodes with respect to the location of the MS. The path of the MS is based on an approximated TSP route that satisfies the length constraint. Only cluster heads $(\mathrm{CH})$ are visited by the MS during its travel. In order to properly address the 'energy holes' that are naturally caused around the $\mathrm{CHs}$ involved in the TSP route, reclustering phases are performed when necessary. Although reclustering is very important, multi-hop communication within the clusters are more energy consuming than single-hop because of multi-hop relays.

In this work, not only a single-hop clustering scheme and reclustering of the network using a fast and energy efficient algorithm is considered, but also a data communication technique for which the communication between the MS and the sensor is highly reliable is employed.

\section{PRELIMINARIES}

In this section the problem to be solved, the system model and the main concepts (system model and circumference visit method) used for resolving the problem are described.

\subsection{Problem description}

Conventional WSN architectures are based on the assumption that the network is dense, so that any two nodes can communicate with each other through multi-hop paths [3]. Yet, this is not always the case.

The sensor nodes cooperatively sense various events in their surrounding environment and report back to the sink [3,5]. In wireless sensor network with static sink, the concentration of data traffic towards the sink causes the nearby nodes to deplete their energy quicker than other nodes, which leaves the sink stranded and disrupts the sensor data reporting. In order to mitigate this problem the usage of mobile sinks was introduced. Mobile sinks implicitly provide load-balancing and help achieving uniform energy-consumption across the network. However, a good number of design requirements and challenges associated with the problem of mobile sink routing in WSN need to be addressed carefully so as to avoid an overhead in terms of energy consumption and packet delays. This work is focused on two of such properties: the selection/location of target nodes and the nearness of the mobile sink to the target nodes. Indeed, in order to minimize the total round trip distance, the mobile sink should visit some target nodes and not all the nodes in the network. These target nodes can be cluster heads $(\mathrm{CH})$ and are identified to hold data from their neighboring nodes and from whom the mobile sink shall collect during subsequent visits. The identification of these nodes should not only take into account residual energy and coverage but also time. This operation will also help in reducing data redundancy and data aggregation. On the other hand, the nearness of the mobile sink to the target nodes during visits determines the reliability of the communication and also energy required by the node to transmit data to the mobile sink.

Based on these properties mobile sink routing constitutes an interesting research field with major requirements. This paper addresses the problem of mobile sink routing within a heterogeneous sensor network with the above mentioned requirements. The sensors nodes are heterogeneous with variable communication ranges. In order to provide an optimal path for the mobile sink, an efficient clustering algorithm to select and determine the locations of the target nodes along the tour path of the mobile sink is used. A definitive and detailed categorization is made and the protocols' advantages and drawbacks are determined with respect to their target applications.

\subsection{Network and Sensing model}

We consider a set of $\mathrm{N}$ sensors nodes randomly dispersed into a given field and following a stochastic distribution [11]. We assume the following properties about the sensor network:

- The field of interest is known and obstacle free. For simplicity in analysis, let's assume that the area is a square in the plane

- The sensor nodes are heterogeneous i.e. they have different communication ranges.

- The network is a typical one for sensor network applications with static nodes.

- The $\mathrm{N}$ sensor nodes are quasi-stationary with similar capabilities (processing / communication), and equal significance. This motivates the need for extending the lifetime of every sensor

- $\quad$ Links are symmetric, i.e., two nodes $v 1$ and $v 2$ can communicate using the same transmission power level.

- The sensor nodes are location-aware, i.e. equipped with GPS-capable antennae.

- Nodes are left unattended after deployment. Therefore, battery re-charge is not possible. Efficient, energy-aware sensor network protocols are thus required for energy conservation.

- Each node has a fixed number of transmission power levels. An example of such sensor nodes are Berkeley Motes.

Once deployed into the given area, the sensor nodes monitor their surrounding environment in order to detect subsequent events that may occur. Thus, the sensors periodically sense the data from this environment, process it and wait for the mobile sink to collect during its visits.

\subsection{The ICP protocol}

ICP is an instantaneous clustering protocol for wireless sensor networks that groups sensor nodes into single-hop clusters in a parallel manner [10]. This protocol considers both time and energy efficiency to determine cluster heads locally while minimizing the total amount of transmissions within the network. Thus the main features of ICP are fast and low cost 
(in terms of number of transmissions and energy consumption) contrary to existing methods which require a long duration on cluster head voting. On the one hand, the protocol minimizes total energy by making use of preassigned probabilities and actual state of nodes to locally determine cluster heads. In addition, only the candidate cluster heads are allowed to send messages during the clustering period. On the other hand, ICP perform parallel clustering which presents the benefit of reducing the duration of clustering for the entire network to be the duration of organizing only one cluster. As depicted on Figure 2, sensors have three roles in this single-hop cluster scheme namely: cluster head $(\mathrm{CH})$, cluster member $(\mathrm{CM})$, and gateway $(\mathrm{GW})$.

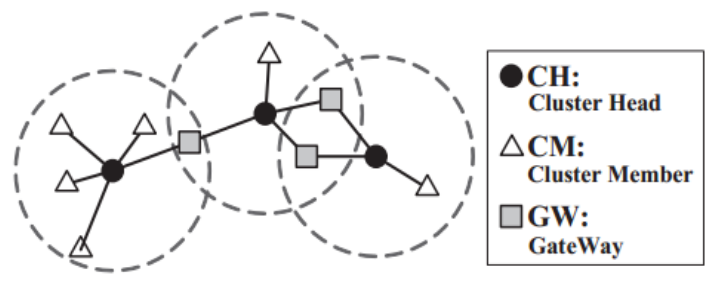

Figure 2: Single-hop cluster topology, source [10]

Moreover complexity analysis carried out in [10] shows that ICP is lightweight with a computational complexity of $\mathrm{O}(1)$ both at $\mathrm{CH}$ side or at $\mathrm{CM} / \mathrm{GW}$ side. This information is obviously verified since a $\mathrm{CH}$ candidate decides to be a $\mathrm{CH}$, $\mathrm{CM}$ or GW based on the number of IDs received from the other $\mathrm{CH}$ candidates. Similarly, ICP reduces both communication overhead and energy consumption as it complexity is of $\mathrm{O}(\mathrm{m})=\mathrm{O}(\log n)$ where $\mathrm{m}$ is the number of cluster heads (derived such as to be sufficient to make the WSN connected by single-hop clustering) and $n$ is the overall number of sensor nodes. Finally ICP is much faster than conventional methods whose time costs are proportional to the density of the network. Rather ICP has a constant time cost of $(\mathrm{T}+\mathrm{D})$ which is the number of time slots used by the clustering scheme.

\subsection{The Circumference visit method}

During regular visits by mobile sink for data collection from CHs, it's important to determine how near the MS will be to the target nodes in order to ensure reliable communication and data transfer. Such a method is the circumference visit presented in [9]. In this case, the path of the MS is designed such as to contact the circumference of the communication circle of each sensor once as shown in Figure 3. Thus, the MS stops regularly at one point on the communication circles of the respective nodes before it continues its trip tour. Considering $\left(\mathrm{x}_{\mathrm{i}}, \mathrm{y}_{\mathrm{i}}\right)$ to be the coordinates of the $\mathrm{i}^{\text {th }}$ point which lies on the circumference of the $i^{\text {th }}$ circle, the point vector $\mathrm{P}$ of these target points is represented in [9] as,

$$
\begin{aligned}
\boldsymbol{P} & =[p(1), p(2), \ldots p(N)] \\
& =\left[\left(x_{1}, y_{1}\right),\left(x_{2}, y_{2}\right), \ldots,\left(x_{N}, y_{\mathrm{N}}\right)\right]
\end{aligned}
$$

Thus, the travel path of the MS is constituted of these p(i) points, forming straight line segments with each other. Likewise the sum $\mathrm{D}$ of these line segments is given in [9] by:

$$
\mathrm{D}=\mathrm{d}(1)+\mathrm{d}(2)+\ldots+\mathrm{d}(\mathrm{N}-1)+\mathrm{d}(\mathrm{N}), \quad \text { for } \mathrm{i}=1 \text { to }(\mathrm{N}-1)
$$

Where

$$
\begin{gathered}
d(i)=\sqrt{ }\left(x_{i}-x_{i+1}\right)^{2}+\left(y_{i}-y_{i_{+1}}\right)^{2}=\|p(i)-p(i+1)\| \\
d(\mathrm{~N})=\sqrt{ }\left(x_{\mathrm{N}}-x_{1}\right)^{2}+\left(y_{\mathrm{N}}-y_{1}\right)^{2}=\|p(\mathrm{~N})-p(1)\|
\end{gathered}
$$

Similarly, it's important to establish a secured session between the communicating peers during MS visits. An authenticated key agreement scheme will be suitable at this stage not only to establish an encryption key between the $\mathrm{CH}$ and mobile sink, but also to authenticate themselves to each other. This encryption key will then be used to encrypt/decrypt information being transmitted from one node the other in order to provide confidentiality and integrity [16].

The sensor nodes on Figure 3 are considered to be the cluster heads (target/visit points).

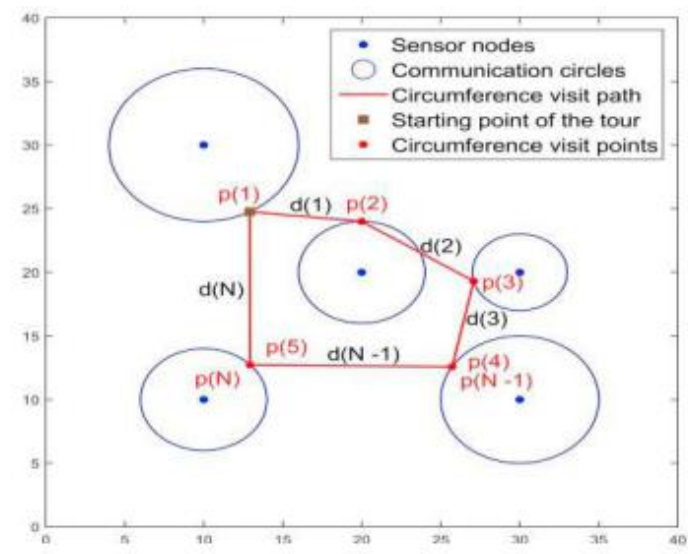

Figure 3: Circumference visit, source [9]

\section{PROPOSED SOLUTION}

In this section, the proposed approach is presented. The proposed system consists of three (02) modules namely identification of visit points and path determination by mobile sink. This section describes each module in detail.

First, the parameters used in the clustering process are defined. Second, the protocol design and pseudo-code are presented. Finally, it's proven that the protocol meets its requirements.

\subsection{Assumptions}

We make the following assumptions: the sensing field is obstacle free, but in case there could be any they should be fixed and known, in which case the patrolling approach presented in [15] could be used in order to avoid these obstacles. Sensor nodes are location-aware, i.e. equipped with a mechanism enabling them to get their position. The sensor nodes are heterogeneous with different communication ranges $r_{i}$, where $i \epsilon\{1,2, \ldots, N\}$ and $N$ the total number of sensor nodes. In this case, two nodes $\mathrm{i}$ and $\mathrm{j}$ are connected if their Euclidean distance is no more than $\min \left(r_{i}, r_{j}\right)$. Before deployment, the nodes are pre-assigned with probability $\mathrm{P}_{\mathrm{CH}}$ as indicated in [10], on the basis of which cluster heads will be determined. According to ICP, this probability is calculated $\mathrm{P}_{\mathrm{CH}}=\beta \mathrm{m} / \mathrm{N}$, where $\beta$ is a constant coefficient (called the redundancy coefficient) and $\mathrm{m}$ the estimated number of cluster heads (called the expectation number). One last assumption is that one or more sinks can be used to visit the target points.

With all this set, sensor nodes can now be deployed into the given field.

\subsection{Identification of visit points}

Once deployed the sensor nodes start to self-organize by executing synchronously the single-hop clustering algorithm in [10] in order to group themselves into connected clusters. After the $\mathrm{T}+\mathrm{D}$ time slots provided by this algorithm, the $\mathrm{m}$ 
expected cluster heads are known. Indeed, any sensor node with probability $\mathrm{P}_{\mathrm{CH}}$ becomes a $\mathrm{CH}$ candidate. Then, upon transmitting their ID messages and based on the number of messages received, a $\mathrm{CH}$ candidate either contends to be $\mathrm{CH}$ or becomes $\mathrm{CM} / \mathrm{GW}$ by an abdication mechanism. These operations are carried out by performing the two procedures presented in [10]. In addition to the pre-assigned probability, the residual energy of sensors is considered as a major factor. For example, a $\mathrm{CH}$ candidate with high residual energy has more chance to transmit its ID earlier than others.

After this operation, the $\mathrm{CH}$ nodes are used as visit points by the mobile sink. The information about $\mathrm{CH}$ is broadcasted to all its closest $\mathrm{CHs}$ in the network and to the sink. The mobile sink then uses the $\mathrm{CH}$ node information to determine its target points for data collection.

Given that the clustering process can be executed each time the mobile sink has to visit sensor in order to collect sensory data, this stage can be repeated as many times as needed without fear of much depleting network resources as it is fast and energy efficient.

\subsection{Path determination}

The path followed by the mobile sink is constituted of the visit points identified after the clustering process. This path is determined by the MS and the corresponding visits points are obtained using the principle of linked lists. Each time the MS needs to visit the sensor nodes, it broadcasts a request (Loc_Req) to determine the location of the closest visit point to it. The closest $\mathrm{CHs}$ which receive this request reply (LocRep) with their location coordinates and communication range r. As illustrated on Figure 4, these closest $\mathrm{CHs}$ also send the
Loc_Req to their own closest CHs. Obviously these requests are forwarded through subsequent GWs nodes. Thus, each $\mathrm{CH}$ receiving the request from another $\mathrm{CH}$, responds with its location information and its value of $r$, then it also receives the same information from the sender $\mathrm{CHs}$. The information about $\mathrm{CH}$ nodes is distributed to all the other $\mathrm{CHs}$ in the network but only neighbor $\mathrm{CHs}$ know themselves. The mobile sink uses the $\mathrm{CH}$ nodes information, i.e. coordinates of each $\mathrm{CH}(\mathrm{i})$ and $\mathrm{r}_{\mathrm{i}}$, to determine its target points for data collection points. Let $\mathrm{P}\left(\mathrm{x}_{\mathrm{Ti}}, \mathrm{y}_{\mathrm{Ti}}\right)$ be a target point found on the communication circle of $\mathrm{CH}(\mathrm{i})$ whose location coordinates are $\left(\mathrm{x}_{\mathrm{i}}, \mathrm{y}_{\mathrm{i}}\right)$, then we have:

$$
\left(x_{\mathrm{Ti}}-x_{i}\right)^{2}+\left(y_{\mathrm{Ti}}-y_{i}\right)^{2}=\mathrm{r}_{\mathrm{i}}^{2}
$$

The mobile sink then starts at the cartesian coordinate $\left(\mathrm{x}_{\mathrm{T} 1}\right.$, $\mathrm{y}_{\mathrm{T} 1}$ ) of its closest visit point $\mathrm{CH}_{1}$ and chooses it movement towards the next $\mathrm{CH}$ indicated by $\mathrm{CH}_{1}$. The movement of the MS on the path follows the principle of linked lists given that the information on the visit points is determined locally and their location information is known only by the neighbor nodes. As shown on Figure 4, the MS only receives the information of the closest $\mathrm{CH}$ to it. Each $\mathrm{CH}(\mathrm{k}), 1<=\mathrm{k}<=\mathrm{N}$, receives from $\mathrm{CH}(\mathrm{k}+\mathrm{t}), 1<=\mathrm{t}<=\alpha$ where $\alpha$ is the number of neighbor $\mathrm{CHs}$ to $\mathrm{CH}(\mathrm{k})$. Thus, it impacts less on the energy consumption of sensor nodes. Moreover, these information changes periodically as the cluster head function rotates. The sink moves toward data collection points (target points) as shown in Figure 3. In order for the MS to be able to return to a previous visit point, each $\mathrm{CH}(\mathrm{k}), 1<=\mathrm{k}<=\mathrm{N}$, sending its information to any of its neighbors receives in turn their own information from each of them.

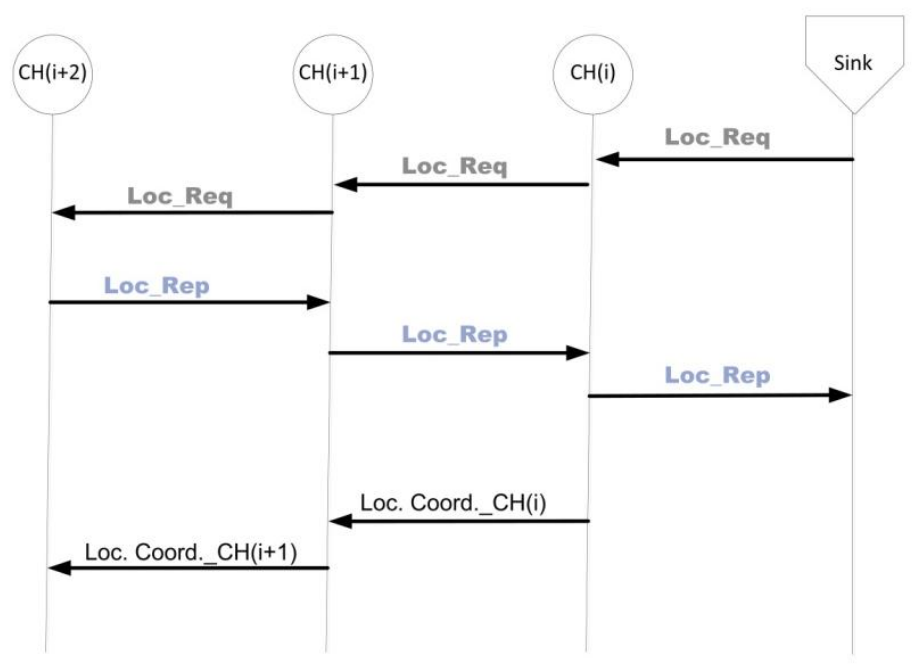

Figure 4: Path determination by mobile sink

\section{PERFORMANCE ANALYSIS}

In order to analyze the performance of the proposed scheme, that of its main components are evaluated, namely the ICP protocol and the circumference visit method. ICP has already been presented in section 3.3 as a fast and energy efficient clustering protocol for WSN. Its complexity analysis reveals that ICP is lightweight with a computational complexity of $\mathrm{O}(1)$ both at $\mathrm{CH}$ side or at $\mathrm{CM} / \mathrm{GW}$ side. This information is obviously verified since a $\mathrm{CH}$ candidate decides to be a $\mathrm{CH}$, $\mathrm{CM}$ or GW based on the number of IDs received from the other $\mathrm{CH}$ candidates. Similarly, ICP reduces both communication overhead and energy consumption as it complexity is of $\mathrm{O}(\mathrm{m})=\mathrm{O}(\log n)$ where $\mathrm{m}$ is the number of cluster heads (derived such as to be sufficient to make the WSN connected by single-hop clustering) and $n$ is the overall number of sensor nodes. Finally ICP is much faster than conventional methods whose time costs are proportional to the density of the network. Rather ICP has a constant time cost of $(\mathrm{T}+\mathrm{D})$ which is the number of time slots used by the clustering scheme.

Moreover, the use of a mobile agent that moves closer to the nodes helps conserve energy since data is transmitted over fewer hops, thus reducing the number of transmitted packets. Also the extra energy spent for the operation and movement 
of the sink does not affect overall sensor network lifetime since the mobile sink is considered an external to the network factor.

The sink moves from one visit point to other by determining a target point where to stop before data transfer can be performed. These target point are found on the circumference of the circles representing the communication region of various sensors. Visit points $\mathrm{p}(1), \mathrm{p}(2), \ldots, \mathrm{p}(\mathrm{N})$ and distances $\mathrm{d}(1), \mathrm{d}(2), \ldots, \mathrm{d}(\mathrm{N}-1), \mathrm{d}(\mathrm{N})$ form the travel polygon as shown in Figure 3. The objective is to minimize the sum of the distances D given in section 3.4, subjected to the constraint restricting the visit points to lie on the circumferences of the circle. In addition, visit points information are not relayed over the entire network but only to the neighboring nodes.

\section{CONCLUSION AND FUTURE WORK}

This work is focused on path discovery scheme for mobile sink based wireless sensor networks using a reliable and low cost method. In this paper a reliable and efficient path discovery method for mobile sink in WSN with heterogeneous sensor nodes (i.e. having variable communication ranges) has been presented. The proposed scheme employs well known protocols from the literature. Given that sink mobility achieves great success in network life time improvement in WSN, clustering is used in order to self-organize the sensor nodes such that MS visits only cluster heads. Then, because the clustering process can be executed each time the mobile sink has to visit sensor in order to collect sensory data, a single-hope clustering technique is used leveraging the limited network resources. The cluster heads are determined locally in a parallel manner, based on pre-assigned probability, resulting in a fast and energy efficient operation. The path of the MS is determined by using the information of the CHs. For this, the circumference visit method in which the MS stops at a point on the communication circle of the sensor nodes before realizing data transfer is used. With the performance analysis performed, we show that the proposed scheme minimizes total round trip of MS and increases network lifetime, by reducing the amount of data transmissions and time consumption of clustering.

\section{REFERENCES}

[1] Yick, J., Mukherjee, B., and Ghosal, D. (2008). Wireless sensor network survey. Computer Networks; 52(12):2292-330.

[2] Yacine, C. (2008) Wireless Sensor Networks [online]. https://moodle.utc.fr/file.php/498/SupportWeb/co/RCSF _web.html (Accessed 19 January 2020).

[3] Francesco, M.D., Das, S.K., and Anastasi, G. (2011). Data Collection in Wireless Sensor Networks with Mobile Elements: A Survey. TOSN, 8, 7:1-7:31.

[4] Shu, Y. et al. (2014). Internet of Things: Wireless Sensor Networks [White paper]. Retrieved July 27, 2019 from Multimedia and print tools for the IEC (International Electrotechnical Commission) community: https://basecamp.iec.ch/download/iec-white-papersinternet-of-things-wireless-sensor-networks/

[5] Ghani, A, Mansoor, K, Mehmood, S, Chaudhry, SA, Rahman, AU, Najmus Saqib, M. (2019). Security and key management in IoT-based wireless sensor networks:
An authentication protocol using symmetric key. Int $\mathrm{J}$ $\begin{array}{llll}\text { Commun } & \text { Syst. } & \text { 2019; }\end{array}$ https://doi.org/10.1002/dac.4139

[6] Li, T., Yuhui, Z., and Zhou, T. (2017). Efficient Anonymous Authenticated Key Agreement Scheme for Wireless Body Area Networks. Security and Communication Networks. 2017. $10.1155 / 2017 / 4167549$.

[7] Renold, A.P., and Athi, B.G. (2019). Energy efficient secure data collection with path-constrained mobile sink in duty-cycled unattended wireless sensor network. Pervasive and Mobile Computing, 55, 1-12.

[8] Gowrishankar, S., Gangaraju, B. T., Manjaiah, D. H., and Sarkar, S. K. (2008). Issues in Wireless Sensor Networks. In Proceedings of the World Congress on Engineering (WCE) Vol I, July 2 - 4, London, U.K.

[9] Thomas, S. and Mathew, T. (2018). Intelligent Path Discovery for a Mobile Sink in Wireless Sensor Network, Procedia Computer Science, Volume 143, pp 749-756, ISSN 1877-0509.

[10] Kong, L., Xiang, Q., Liu, X., Liu, X.-Y., Gao, X., Chen, G. and Wu, M.-Y. (2016). ICP: Instantaneous Clustering Protocol for Wireless Sensor Networks. Computer Networks. 101. 10.1016/j.comnet.2015.12.021.

[11] Kong, L., Zhao, M., Liu, X.-Y., Lu, J., Liu, Y., Wu, M.Y., Shu, W. (2014b). Surface coverage in sensor networks, IEEE Trans. Parallel Distrib. Syst. 25(1) 234 243

[12] Tunca, C., Isik, S., Donmez, M.Y., \& Ersoy, C. (2014). Distributed Mobile Sink Routing for Wireless Sensor Networks: A Survey. IEEE Communications Surveys \& Tutorials, 16, 877-897.

[13] Thomas, S. and Mathew, T. (2019). Minimum Bend Shortest Rectilinear Route Discovery for a Moving Sink in a Grid Based Wireless Sensor Network. INTL JOURNAL OF ELECTRONICS AND TELECOMMUNICATIONS, VOL. 65, NO.2 , PP. $267-$ 276.

[14] Mamalis, B. G. (2014). Prolonging Network Lifetime in Wireless Sensor Networks with Path-Constrained Mobile Sink. International Journal of Advanced Computer Science and Applications (IJACSA), Vol. 5, No. 10.

[15] Elie, T. F., Emmanuel, T., Fabrice, L. \& Abderrafiaa, K. (2014). Multi-agent Patrolling: Multi-Objective Approach of the Event Detection by a Mobile Wireless Sensors Network. International Journal of Computer Applications (0975 8887) Volume 88 - No. 12, February.

[16] Aline, Z. T., Elie, T. F., Emmanuel, T. and Adnen, E. A. (2019). Peer to Peer Authentication for Index-based Distributed Data Collection: A Zero-Knowledge-based Scheme to Security for Wireless Sensor Networks. American Journal of Advanced Research, 3-2, pages 1-6, doi: 10.5281/zenodo.3368588.

[17] L. Kong, M. Zhao, X.-Y. Liu, J. Lu, Y. Liu, M.-Y. Wu, W. Shu, (2014b). Surface coverage in sensor networks, IEEE Trans. Parallel Distrib. Syst.25 (1) 234-243. 